\title{
Aplicação de resíduos da agroindústria e agropecuária na cultura de Raphanus sativus $L$.
}

Os resíduos gerados pela agroindústria e agropecuária podem poluir o ambiente quando descartados de maneira incorreta. A reutilização destes resíduos é uma alternativa sustentável possibilitando um equilibro ambiental, econômico e social para o planeta. Este trabalho visou avaliar as diferentes doses de cama de frango e resíduos de Stevia rebaudiana (Bert.) Bertoni na cultura de Raphanus sativus L. (rabanete). Realizou-se um experimento em vasos com delineamento inteiramente casualizado com seis tratamentos, contendo compostos orgânicos que foram: (T1) latossolo vermelho, (T2) 100\% do resíduo de S. rebaudiana, (T3) 100\% de cama de frango, (T4) $75 \%$ do resíduo de S. rebaudiana e $25 \%$ de cama de frango, (T5) 50\% do resíduo de S. rebaudiana e 50\% de cama de frango e (T6) $25 \%$ do resíduo de S. Rebaudiana e $75 \%$ de cama de frango, com 10 repetições cada tratamento. A avaliação foi realizada 25 dias após o plantio das mudas de rabanete. As variáveis avaliadas foram os parâmetros biométricos como altura da planta, comprimento da raiz, número de folhas, área foliar e peso da matéria seca. A análise química do resíduo de $\mathrm{S}$. rebaudiana apresentou uma quantidade considerável de potássio, indicando a presença de um nutriente importante para a cultura do rabanete. Os rabanetes cultivados com $100 \%$ do resíduo de S. rebaudiana (T2), apresentaram um crescimento e desenvolvimento da parte aérea e das raízes maiores em relação aos outros tratamentos. Já as plantas que foram cultivadas em concentrações maiores de $50 \%$ de cama de frango morreram no início do desenvolvimento. A utilização deste resíduo agroindustrial $\mathrm{S}$. rebaudiana, sugeriu benefícios à cultura do rabanete e, também, como uma forma correta de destinação deste composto no ambiente.

\section{Application of agribusiness and agriculture residues in the culture of Raphanus sativus $L$.}

The waste generated by agribusiness and agriculture can pollute the environment when disposed of incorrectly. The reuse of this waste is a sustainable alternative, enabling an environmental, economic and social balance for the planet. This work aimed to evaluate the different doses of chicken litter and Stevia rebaudiana (Bert.) Bertoni residues in the culture of Raphanus sativus L. (radish). An experiment was carried out in pots with a completely randomized design with six treatments, containing organic compounds which were: (T1) red latosol, (T2) 100\% of the residue of S. rebaudiana, (T3) $100 \%$ of chicken litter, ( T4) $75 \%$ of the residue of S. rebaudiana and $25 \%$ of chicken litter, (T5) $50 \%$ of the residue of S. rebaudiana and $50 \%$ of chicken litter and (T6) $25 \%$ of the residue of S. rebaudiana and $75 \%$ of chicken litter, with 10 repetitions each treatment. The evaluation was carried out 25 days after planting the radish seedlings. The variables evaluated were biometric parameters such as plant height, root length, number of leaves, leaf area and dry matter weight. The chemical analysis of the $\mathrm{S}$. rebaudiana residue showed a considerable amount of potassium, indicating the presence of an important nutrient for the culture of the radish. Radishes grown with $100 \%$ of the $S$ rebaudiana residue (T2), showed a growth and development of the aerial part and larger roots in relation to the other treatments. Plants that were grown in concentrations greater than $50 \%$ of chicken litter, on the other hand, died early in development. The use of this agroindustrial residue S. rebaudiana, suggested benefits to the culture of the radish and, also, as a correct way of destination of this compound in the environment.

Keywords: Organic Fertilizer; Sustainable Agriculture; Poultry litter; Radish; Stevia rebaudiana.

Topic: Desenvolvimento, Sustentabilidade e Meio Ambiente

Reviewed anonymously in the process of blind peer
Received: 04/02/2021 Approved: 25/02/2021
Isadora Fernanda Sperandio (iD)

Centro Universitário de Maringá, Brasil

http://lattes.cnpq.br/3359764111970624

http://orcid.org/0000-0001-9287-6576

isadora.sperandio@hotmail.com

Luiz Henrique dos Reis Bocaleti (D)

Centro de Ensino Superior de Maringá, Brasil

http://lattes.cnpq.br/9185284432984159

http://orcid.org/0000-0003-0034-9280

bocaletiluizh@hotmail.com

Francielli Gasparotto id

Centro Universitário de Maringá, Brasil

http://lattes.cnpq.br/2673470812353146

http://orcid.org/0000-0002-4038-7364

francielli.gasparotto@unicesumar.edu.br

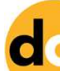

DOI: 10.6008/CBPC2179-6858.2021.002.0046
Edneia Aparecida de Souza Paccola (iD Centro Universitário de Maringá, Brasil

http://lattes.cnpq.br/5090759600495959

http://orcid.org/0000-0002-3182-3224

edneia.paccola@gmail.com

\section{Referencing this:}

SPERANDIO, I. F.; BOCALETI, L. H. R.; GASPAROTTO, F.; PACCOLA, E. A. S.. Aplicação de resíduos da agroindústria e agropecuária na cultura de Raphanus sativus L.. Revista Ibero Americana de Ciências Ambientais, v.12, n.2, p.539-547, 2021. DOI: http://doi.org/10.6008/CBPC21796858.2021 .002 .0046 


\section{INTRODUÇÃO}

Os resíduos gerados pela agroindústria têm causado preocupação, pois, muitas vezes, são descartados de forma incorreta. A busca por alternativas, como a reutilização destes resíduos na agricultura, é uma possibilidade interessante no ponto de vista econômico, ambiental e social (PIRES et al., 2008).

Neste sentido, Bonfim-Silva et al. (2013) e Marques et al. (2018) destacam as vantagens do uso de resíduos industriais e a compostagem na produção vegetal, diminuindo a redução de fertilizantes do solo e proporcionando uma destinação correta destes resíduos.

Com a utilização de adubos orgânicos, podem ser observados vários benefícios, tendo efeito em propriedades físicas, químicas, biológicas e físico-química do solo (HIGASHIKAWA et al., 2016; TRANI et al., 2013; LANDGRAF et al., 2005).

Penteado (2007) e Malavolta et al. (2002) ressaltam que a utilização de resíduos vegetais e esterco proporciona maior resistência e saúde às plantas, que vão sendo enfraquecidas pelo uso constante de adubação química.

Outros estudos destacam o desenvolvimento de olerícolas na presença de resíduos, como casca de ovo, resíduo da secagem do grão de café, fibra de coco do caroço e palmito do açaí, esterco bovino e cama de frango (KRAUSE et al., 2017; CORREA et al., 2019; ALVES et al., 2017; PELÁ et al., 2017).

Com o consequente aumento das atividades avícolas, houve a possibilidade do aproveitamento dos dejetos das aves (cama de frango) como fonte de nutrientes (MENEZES et al., 2008). Quando manejada adequadamente, a cama de frango pode suprir parcial ou totalmente o fertilizante químico (BLUM et al., 2003).

Além da cama de frango, outro resíduo resultante do processo agroindustrial na região noroeste do Paraná é o caule e folhas de Stevia rebaudiana, pouco explorado como fonte de nutrientes no solo para produção de olerícolas. Ao ser processada, a planta de estévia gera um subproduto resultante de um processo de extração dos edulcorantes das folhas de Stevia rebaudiana Bertoni, denominado de caramelo (MIOTTO et al., 2004).

Acredita-se que os resíduos oriundos da avicultura de corte, como a cama de frango, e da indústria de edulcorantes, como a S. rebaudiana, possam ser alternativas sustentáveis na produção do rabanete $(R$. sativus L.). O rabanete é uma cultura olerácea de ciclo curto, no qual o ambiente exerce grande interferência na produção de suas raízes (FILGUEIRA, 2008).

O nível de conscientização quanto às relações da agricultura com o meio ambiente e os recursos naturais vem crescendo substancialmente. Diante disso, produtores passaram a empregar práticas agroecológicas na produção de vegetais (SILVA et al., 2017), o que passou a valorizar o homem no campo, sua família, trabalho e cultura (AVILA et al., 2007).

O presente trabalho teve como objetivo analisar as melhores formas de se reutilizar os compostos orgânicos do resíduo de Stevia rebaudiana e de cama de frango, avaliando o crescimento, produtividade e qualidade das raízes de rabanete. 


\section{MATERIAS E MÉTODOS}

O experimento foi conduzido em estufa de lona de plástico transparente na Instituição de Ensino Superior (IES) em Maringá - Paraná. Cujas coordenadas são 515' $01^{\prime \prime}$ de longitude norte e $23^{\circ} 26^{\prime} 21^{\prime \prime}$ de latitude sul, estando em uma altitude de $525 \mathrm{~m}$ em relação ao nível do mar.

A compostagem de cama de frango foi cedida por uma granja, sediada no município vizinho. A compostagem foi retirada de um galpão utilizado para criação de frangos de corte. Já os resíduos de plantas de S. rebaudiana foram cedidos por uma empresa de adoçante de estévia no município.

A compostagem obtida pelos resíduos de plantas de Stevia rebaudiana teve sua decomposição completa aos 75 dias após o enleiramento e quatro revolvimentos da pilha de compostagem manualmente para melhor aeração.

Os dois compostos foram misturados nas seguintes proporções: Tratamento 1 (T1): testemunha (latossolo vermelho); Tratamento 2 (T2): 100\% do composto de Stevia rebaudiana; Tratamento 3 (T3): 100\% do composto de cama de frango; Tratamento 4 (T4): 75\% do resíduo de Stevia rebaudiana e 25 \% de cama de frango; Tratamento 5 (T5): 50\% do resíduo de Stevia rebaudiana e 50\% de cama de frango; Tratamento 6 (T6): $25 \%$ do resíduo de Stevia rebaudiana e $75 \%$ de cama de frango.

Após cinco dias, as mudas de rabanete foram colocas nos vasos de 2,5 L, para cada tratamento foram realizadas 10 repetições. A cultura foi irrigada diariamente com $200 \mathrm{ml}$ de água, após 25 dias de plantio, os rabanetes foram retirados dos vasos e levados ao laboratório de solos da IES para coleta de dados.

Inicialmente, foram avaliados os parâmetros biométricos, como a altura da planta, a distância do colo da planta até o ápice caulinar; o comprimento da raiz e o diâmetro da raiz (paquímetro digital 150mm). 0 número de folhas foi definido como o somatório das folhas de cada exemplar. O peso da matéria seca foi determinado como sendo o peso da raiz e das folhas após a permanência em estufa por 48 horas, na temperatura de $65-85^{\circ} \mathrm{C}$.

O delineamento experimental foi o inteiramente casualizado com arranjo fatorial $6 \times 2$, com 10 repetições, os tratamentos aplicados nos vasos continham diferentes combinações de cama de frango e resíduo de Stevia rebaudiana. Os dados encontrados foram analisados estatisticamente por meio de análise de variância (ANOVA) pelo teste $F$, e as médias comparadas pelo teste de Tukey a $5 \%$ de probabilidade, para desenvolver os gráficos os dados foram rodados no programa Statistica 7.1. e elaborados no padrão box plot.

\section{RESULTADOS E DISCUSSÃO}

A caracterização química dos resíduos de cama de frango e Stevia rebaudiana estão apresentados nas Tabelas 1 e 2, respectivamente. As plantas dos tratamentos T3, T5 e T6 não foram capazes de se desenvolver, possivelmente devido ao grande teor de nutriente presente na cama de frango, a qual apresentou 2,39\%; 0,18\% e 6,93\% de $\mathrm{N}$ total, $\mathrm{P}$ e K, respectivamente (Tabela 1). 
A cama de frango apresenta uma alta concentração de macronutrientes NPK (nitrogênio, fósforo, potássio) (GIROTTO et al., 2003). Também apresenta em sua composição cálcio e magnésio, sendo rica em matéria orgânica, o que favorece maior absorção de água e nutrientes (JULIANO et al., 2019).

Tabela 1: Resultados analíticos das características químicas dos macronutrientes e micronutrientes da cama de frango utilizada nos vasos do experimento.

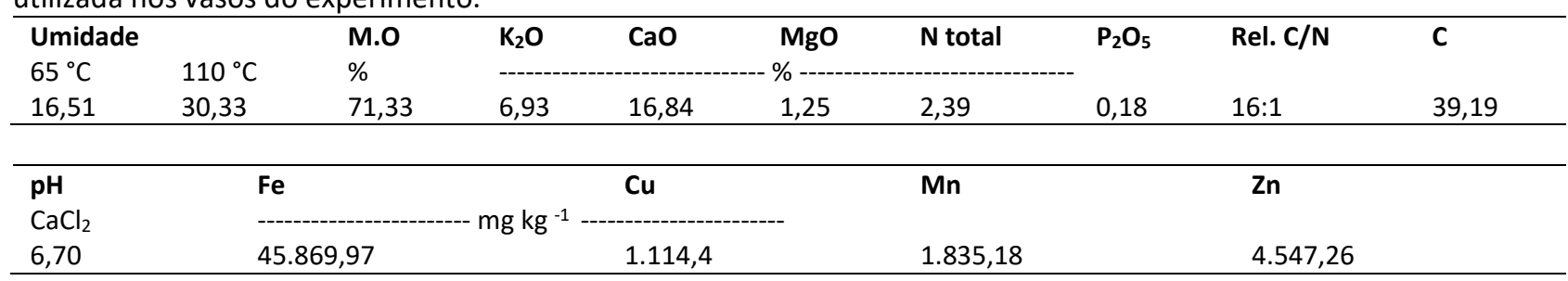

Fonte: Adaptado de Análise Química (método de Kjeldahl) realizada no Laboratório Rural de Maringá (2018).

A análise química do resíduo de S. rebaudiana apresentou um total de 1,37\% de $\mathrm{K}_{2} \mathrm{O}$ (Tabela 2), indicando a presença de um nutriente importante para a cultura do rabanete.

Tabela 2: Resultados analíticos das características químicas dos macronutrientes e micronutrientes da compostagem de Stevia rebaudiana

\begin{tabular}{|c|c|c|c|c|c|c|c|c|c|}
\hline \multicolumn{2}{|l|}{ Umidade } & M.O & $\mathrm{K}_{2} \mathrm{O}$ & $\mathrm{CaO}$ & MgO & $\mathrm{N}$ total & $\mathrm{P}_{2} \mathrm{O}_{5}$ & \multirow[t]{2}{*}{ Rel. C/N } & \multirow[t]{2}{*}{ C } \\
\hline $65^{\circ} \mathrm{C}$ & $110^{\circ} \mathrm{C}$ & $\%$ & ---- & & $--\%$ & ------- & & & \\
\hline 35,88 & 64,81 & 89.04 & 1,37 & 5,63 & 1,21 & 1,38 & 0,80 & $35: 1$ & 48,92 \\
\hline pH & \multicolumn{2}{|c|}{$\mathrm{Fe}$} & \multirow{2}{*}{\multicolumn{3}{|c|}{$\mathrm{Cu}$}} & $M n$ & \multicolumn{3}{|c|}{$\mathrm{Zn}$} \\
\hline $\mathrm{CaCl}_{2}$ & & -1--- & & & & & & & \\
\hline 3,44 & \multicolumn{2}{|c|}{$10.468,05$} & \multicolumn{3}{|c|}{563,46} & 1.96 & \multicolumn{3}{|c|}{602,16} \\
\hline
\end{tabular}

Fonte: Adaptado de Análise Química (método de Kjeldahl) realizada no Laboratório Rural de Maringá (2018).

Maia et al. (2011) afirmam que o potássio é muito importante para o rabanete, se destacando em relação aos demais nutrientes. Castro et al. (2016) recomendam a adubação potássica para aumento da produtividade do rabanete. Maia et al. (2011) também ressaltam a importância da adubação potássica no rabanete, tendo um incremento de $91,5 \%$ na massa fresca da raiz quando comparado a um cultivo sem adubação.

O fósforo, um dos principais nutrientes limitantes à produtividade da cultura, está envolvido em processos metabólicos, como transferência de energia, síntese proteica, fotossíntese e respiração (PANDEY et al., 2015).

Resultados semelhantes foram obtidos por Rodrigues et al. (2013) para o diâmetro da raiz com adubação orgânica em solos com teores adequados de P e K e por Castro et al. (2016) com adubação potássica.

Quando ocorrem problemas nutricionais, principalmente os relacionados com o potássio e o nitrogênio, dificilmente são possíveis serem corrigidos durante o ciclo (EL-DESUKI et al., 2005).

Em culturas que acumulam reservas, como os carboidratos nas raízes, a qualidade nutricional varia de acordo com os teores de potássio (ZÖRB et al., 2014).

A adubação é um fator limitante para a produção, qualidade e desenvolvimento das culturas agrícolas. A ordem de nutrientes extraídos pela cultura, de acordo com Echer et al. (2009), é: $\mathrm{K}>\mathrm{N}>\mathrm{Ca}>\mathrm{P}>\mathrm{S}>\mathrm{Mg}$. 
Chohura et al. (2011) e Baloch et al. (2014) sugerem que a cultura apresenta respostas positivas e diferenciadas a doses crescentes de potássio e nitrogênio. $O$ nitrogênio é o componente essencial de aminoácidos, proteínas, ácidos nucleicos e clorofila e o potássio está envolvido com reações bioquímicas necessárias para o metabolismo das plantas (NURZYŃSKA-WIERDAK et al., 2012).

O uso de doses elevadas de nitrogênio na cultura do rabanete pode proporcionar crescimento excessivo da parte aérea, em razão da translocação e alocação de assimilados nas raízes, o que reduz a produtividade comercial (AQUINO et al., 2006; GRANGEIRO et al., 2007).

O resíduo de $S$. rebaudiana, quando incorporado altas concentrações da cama de frango, também não demonstrou resultados favoráveis à cultura, como observado no tratamento T5 e T6. Se utilizada sem ser estabilizada, a cama de frango pode causar efeitos negativos à cultura, inibindo o crescimento das plantas e gerando prejuízos na produção (HEYLMANN et al., 2013).

Silva et al. (2015), Basha et al. (2015) e Cecílio Filho et al. (1998) relataram que o rabanete não é exigente quanto aos nutrientes presentes no solo.

$\mathrm{O}$ pH verificado nas análises de cama de frango e resíduo de S. rebaudiana foi de 6,7 e 3,3, respectivamente. Indicando um pH favorável ao desenvolvimento das raízes e parte aérea da cultura. $\mathrm{O} p H$ mais indicado para produção de rabanete varia entre 5,5 e 6,8 (FILGUEIRA, 2008).

Ressaltando que os rabanetes cultivados em concentrações acima de $50 \%$ de cama de frango como nos tratamentos (T3) 100\% de cama de frango, (T5) 50\% do resíduo de S. rebaudiana e 50\% de cama de frango e (T6) $25 \%$ do resíduo de S. Rebaudiana e $75 \%$ de cama de frango, no início do desenvolvimento morreram. As avaliações das médias foram realizadas com as plantas que se desenvolveram nos tratamentos (T1) latossolo vermelho, (T2) 100\% do resíduo de S. rebaudiana, (T4) 75\% do resíduo de S. rebaudiana e 25 $\%$ de cama de frango.

As plantas de rabanete que se desenvolveram somente na presença do resíduo de S. rebaudiana, demonstraram resultados positivos em relação à altura e peso da parte aérea, sendo superiores ao tratamento 1 e 4 (Figura 1).
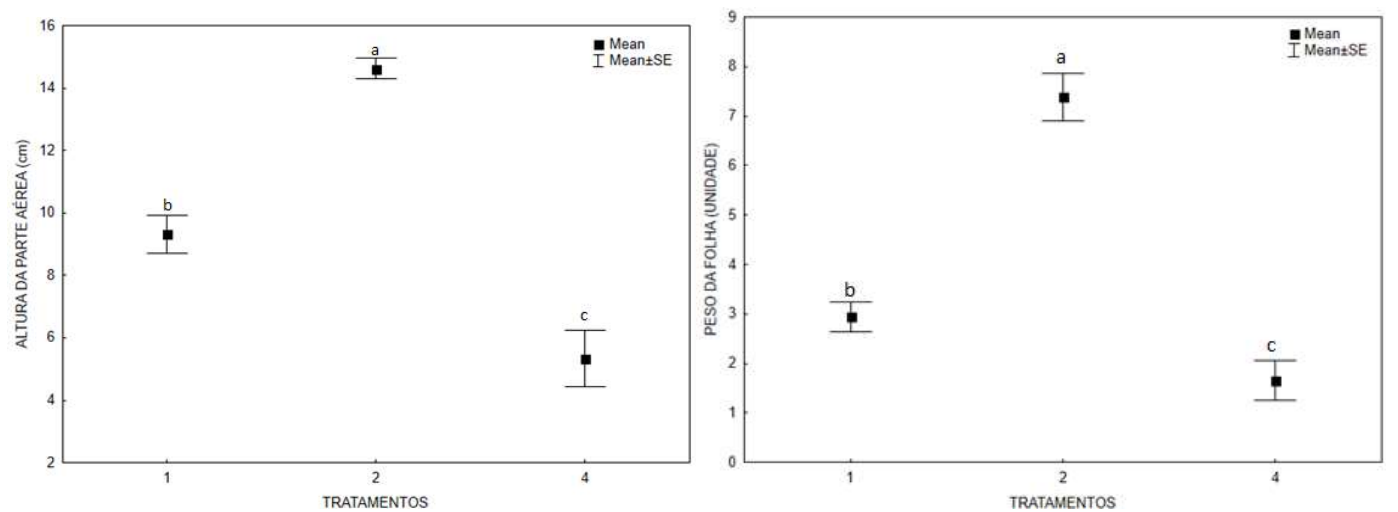

Figura 1: Altura $(\mathrm{cm})$ e peso da parte aérea (gr) de Raphanus sativus L., respectivamente, sob as diferentes doses dos tratamentos T1 (latossolo vermelho), T2 (100\% do composto de Stevia rebaudiana), T4 (75\% do resíduo de Stevia rebaudiana e 25 \% de cama de frango) a que foram submetidos (gráficos no padrão Box plot).

Quanto ao número de folhas, as plantas do tratamento 2 (T2) se destacaram por apresentarem maior número, com uma média de 9,4 folhas por planta (Figura 2). Mas, em comparação com todos os outros 
tratamentos, independente do desenvolvimento da raiz, também apresentaram um número significativo destas, favorecendo a atividade fotossintética da cultura.

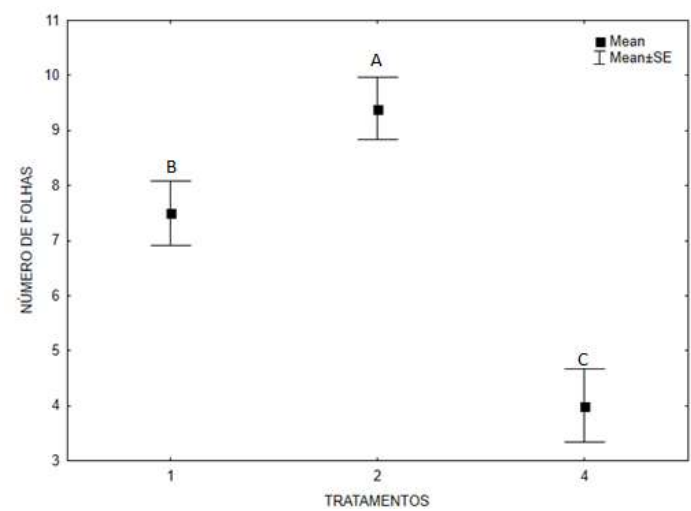

Figura 2: Total de número de folhas de Raphanus sativus L. dos tratamentos T1 (latossolo vermelho) T2 (100\% do composto de Stevia rebaudiana), T4 (75\% do resíduo de Stevia rebaudiana e $25 \%$ de cama de frango) quando tratados com composto de Stevia rebaudiana e cama de frango (Padrão Box plot).

Segundo Linhares et al. (2010), o número de folhas tem grande importância, pois este órgão é o local onde ocorre o processo bioquímico conhecido como fotossíntese, sendo responsável pela grande produção de fotoassimilados que serão enviados e utilizados pelos órgãos produtivos da planta. O desenvolvimento da parte aérea e, consequentemente das raízes, está associado à adequada qualidade e condições físicas que o solo apresenta (FREDDI et al., 2008).

O resíduo de S. rebaudiana apresentou grande destaque, pois é uma excelente fonte de carbono e matéria orgânica, apresentando 48,92\% e 89,04\% respectivamente. Lanna et al. (2018) afirmam que a cultura do rabanete é favorecida pelo alto teor de matéria orgânica, sem a necessidade de nova fertilização.

No tratamento 2, verificou-se maior número de plantas com as raízes rachadas, apesar de apresentarem o maior peso e o maior diâmetro (Figura 3). A quantidade de água utilizada no experimento por vaso $(200 \mathrm{ml})$, demonstrou ser inadequada para as plantas, devido à presença de rachaduras nas raízes.
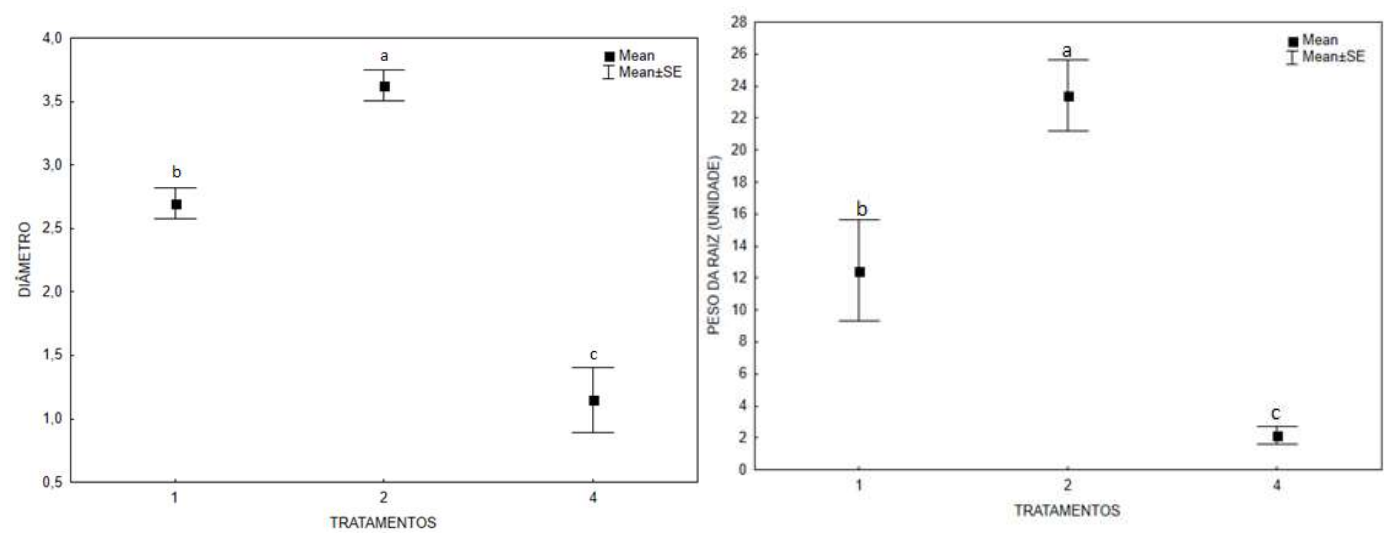

Figura 3: Diâmetro e peso das raízes de Raphanus sativus L., respectivamente, sob as diferentes doses de adubação de cama de frango e S. rebaudiana, T1 (latossolo vermelho), T2 (100\% do composto de Stevia rebaudiana), T4 (75\% do resíduo de Stevia rebaudiana e 25 \% de cama de frango) (Padrão Box plot).

Segundo Filgueira (2008), um dos fatores que podem acarretar rachaduras nas raízes de rabanete são as oscilações hídricas, o que torna a raiz imprópria para o comércio, gerando prejuízo aos agricultores. 
Bregonci et al. (2008) salientam que o estresse hídrico afeta negativamente o diâmetro do bulbo do rabanete. Outro fator que pode ocasionar rachadura nas raízes são temperaturas em torno dos $30 \stackrel{\circ}{ }$, devido à formação de lignina ao redor das células, ocasionadas pelo aquecimento (COSTA et al., 2006).

Nenhum dos tratamentos apresentou raiz com isoporização (raízes esponjosas e insipidas), o que implica numa melhor qualidade e produtividade comercial das raízes, por outro lado, algumas raízes se encontravam rachadas.

Para que as raízes se desenvolvam da melhor maneira possível, são recomendadas temperaturas baixas e dias curtos. A aplicação de adubos orgânicos, que liberam grande quantidade de nitrogênio, também pode vir a ocasionar raízes rachadas e isoporizadas (COSTA et al., 2006), o que não é favorável à cultura, pois prejudica a estética e, consequentemente, a sua comercialização, pois não são aceitas para este fim.

Os rabanetes cultivados na presença dos resíduos de S. rebaudiana (T2) apresentaram o maior peso e comprimento das raízes (Figura 4), evidenciando a eficácia deste resíduo para o desenvolvimento deste órgão vegetal de elevado valor comercial para os produtores.
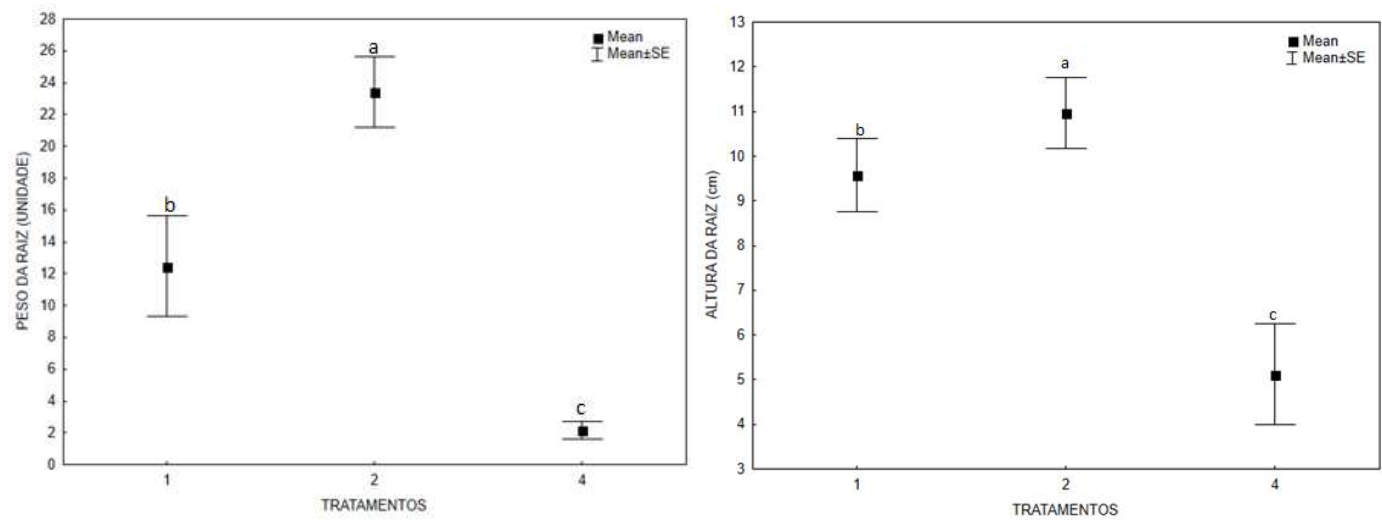

Figura 4: Peso e comprimento das raízes de Raphanus sativus L. em função dos tratamentos T1 (latossolo vermelho), T2 (100\% do composto de Stevia rebaudiana), T4 (75\% do resíduo de Stevia rebaudiana e 25 \% de cama de frango), respectivamente (Padrão Box plot).

As fontes de adubos orgânicos utilizados apresentaram diferentes efeitos nesta cultura (Tabela 3). A cama de frango em concentrações a partir de $25 \%$ não contribuiu para o desenvolvimento das plantas, afetando a formação das raízes, apresentando assim respostas negativas sobre a produtividade total do rabanete.

Tabela 3: Médias da massa da parte aérea (MPA), peso da raiz (PR), comprimento da raiz (CR), número total de folhas (NTF), diâmetro da raiz (DR), peso das folhas (PF) de rabanete obtidas do composto de cama de frango e resíduo de Stevia rebaudiana sobre o crescimento de Raphanus sativus

\begin{tabular}{|l|l|l|l|l|l|l|}
\hline Tratamento & MPA & PR & CR & NTF & DR & PF \\
\hline T1 & $0,35 \mathrm{~b}$ & $9,57 \mathrm{~b}$ & $9,57 \mathrm{~b}$ & $7,50 \mathrm{~b}$ & $2,70 \mathrm{~b}$ & $2,95 \mathrm{~b}$ \\
\hline T2 & $0,90 \mathrm{a}$ & $10,96 \mathrm{a}$ & $10,96 \mathrm{a}$ & $9,40 \mathrm{a}$ & $3,63 \mathrm{a}$ & $7,43 \mathrm{a}$ \\
\hline T4 & $0,28 \mathrm{c}$ & $4,43 \mathrm{c}$ & $5,18 \mathrm{c}$ & $4 \mathrm{c}$ & $1,28 \mathrm{c}$ & $1,7 \mathrm{c}$ \\
\hline Teste F & $44,24^{* *}$ & $22,98^{* *}$ & $11,22^{* *}$ & $22,03^{* *}$ & $11,92^{* *}$ & $54,50^{* *}$ \\
\hline CV \% & 32,76 & 26,37 & 33,22 & 14,1 & 23,85 & 32,04 \\
\hline
\end{tabular}

**Resultado é estatisticamente significante.

Por outro lado, a utilização de resíduo de $S$. rebaudiana, se destacou em relação aos demais tratamentos, incluindo os tratamentos somente com solo. A utilização do resíduo demonstrou ser eficiente 
na produção de folhas, no aumento do peso e diâmetro das raízes, o que representa uma grande importância comercial.

\section{CONCLUSÕES}

A utilização da compostagem dos resíduos de Stevia rebaudiana demonstrou ser favorável à cultura, afetando positivamente o desenvolvimento e produtividade da parte aérea e das raízes de Raphanus sativus L., trazendo benefícios para a cultura do rabanete, não somente no quesito produtividade, como também na geração de uma agricultura sustentável, uma destinação correta deste composto e contribuindo para evitar problemas ambientais.

\section{REFERÊNCIAS}

ALVES, J. C.; PÔRTO M. L. A.; OLIVEIRA, A. F. S.. Níveis de esterco bovino em substratos para produção de mudas de pimenta Malagueta. Revista Craibeiras de Agroecologia, v.1, n.1, p.1-4, 2017.

AQUINO, L. A.; PUIATTI, M.; PEREIRA, P. R. G.; PEREIRA, F. H. F.; LADEIRA, I. R.; CASTRO, M. R. S.. Produtividade, qualidade e estado nutricional da beterraba de mesa em função de doses de nitrogênio. Horticultura Brasileira, Brasília, v.24, n.2, p.199-203, 2006. DOI: http://dx.doi.org/10.1590/S0102$\underline{05362006000200015}$

AVILA, V. S.; ABREU, V. M. N.; FIGUEIREDO, E. A. P.; BRUM, P. A. R.; OLIVEIRA, U.. Valor agronômico da cama de frango após reutilização por vários lotes consecutivos.

Comunicado Técnico Embrapa Suínos e Aves, 46. Concórdia: Embrapa, 2007.

BALOCH, P. A.; UDDIN, R.; NIZAMANI, F. K.; SOLANGI, A. H.; SIDDIQUI, A. A.. Effect of Nitrogen, Phosphorus and Potassium Fertilizers on Growth and Yield Characteristics of Radish (Raphinus Sativus L.). American-Eurasian Journal of Agricultural \& Environmental Sciences, v.14, n.6, p.565-569, 2014. DOI:

https://dx.doi.org/10.5829/idosi.aejaes.2014.14.06.12350

BASHA, D. M. R. A.; EL-AILA, H. I.. Resposta da pulverização foliar com aminoácidos e uso integrado de fertilizantes nitrogenados em plantas de rabanete (Raphanus Sativus). International Journal of ChemTech Research, v.8, p.135140, 2015.

BLUM, L. E. B.; AMARANTE, C. V. T.; GÜTTLER, G.; MACEDO, A. F.; KOTHE, D. M.; SIMMLER, A. O.; PRADO, G.; GUIMARÃES, L. S.. Produção de moranga e pepino em solo com incorporação de cama aviária e casca de pinus. Horticultura Brasileira, v.21, n.4, p.627-631, 2003. DOI: http://dx.doi.org/10.1590/s0102-05362003000400010

BONFIM-SILVA, E. M.; CABRAL, C. E. A.; SILVA, T. J. A.; MOREIRA, J. C. F.; CARVALHO, J. C. S.. Cinza vegetal: características produtivas e teor de clorofila do capimmarandu. Bioscience Journal, Uberlândia, v.29, n.5, p.12151225, 2013.

BREGONCI, I. S.; ALMEIDA, G. D.; BRUM, V. J.; ZINI JUNIOR, A.; REIS, E. F.. Desenvolvimento do sistema radicular do rabanete em condição de estresse hídrico. Idesia, Arica, v.26, n.1, p.33-38, 2008. DOI:

http://dx.doi.org/10.4067/s0718-34292008000100005

CASTRO, B. F.; SANTOS, L. G.; BRITO, C. F. B.; FONSECA, V. A.; BEBÉ, F. V.. Produção de rabanete em função da adubação potássica e com diferentes fontes de nitrogênio. Revista de Ciências Agrárias, v.39, n.3, p.341-348, 2016. DOI: http://dx.doi.org/10.19084/rca15131

CECÍLIO FILHO, A. B.; FAQUIN, V.; FURTINI NETO, A. E.; SOUZA, R. J.. Deficiência nutricional e seu efeito na produção de rabanete. Científica, São Paulo, v.26, n.1-2, p.231-241, 1998.

CHOHURA, P.; KOLOTA, E.. The effect of nitrogen fertilization on radish yielding. Acta Scientiarum Polonorum Hortorum Cultus, v.10, n.1, p.23-30, 2011.

CORREA, B. A.; PARREIRA, M. C.; MARTINS, J. S.; RIBEIRO, R. C.; SILVA, E. M.. Reaproveitamento de resíduos orgânicos regionais agroindustriais da Amazônia Tocantina como substratos alternativos na produção de mudas de alface. Revista Brasileira de Agropecuária Sustentável (RBAS), v.9, n.1, p.97-104, 2019. DOI: http://dx.doi.org/10.21206/rbas.v9i1.7970

COSTA, C. C.; OLIVEIRA, C. D.; SILVA, C. J.; TIMOSSI, P.C.; LEITE, I. C.. Crescimento, produtividade e qualidade de raízes de rabanete cultivadas sob diferentes fontes e doses de adubos orgânicos. Horticultura Brasileira, Vitória da Conquista, v.24, n.1, p.118-122, 2006. DOI: http://dx.doi.org/10.1590/s0102-05362006000100024

ECHER, F. R.; DOMINATO, J. C.; CRESTE, J. E.. Absorção de nutrientes e distribuição da massa fresca e seca entre órgãos de batata-doce. Horticultura Brasileira, v.27, n.2, p.176-182, 2009. DOI: http://dx.doi.org/10.1590/s010205362009000200010

EL-DESUKI, M.; SALMAN, S. R.; EL-NEMR, M. A.; ABDELMAWGOUD, A. M. R.. Effect of Plant Density and Nitrogen Application on the Growth, Yield and Quality of Radish (Raphanus Sativus L.). Journal of Agronomy, v.4, n.3, p.225229, 2005. DOI: http://dx.doi.org/10.3923/ia.2005.225.229 
FILGUEIRA, F. A. R.. Novo manual de olericultura: agrotecnologia moderna na produção e comercialização de hortaliças. 2 ed. Viçosa: UFV, 2008.

FREDDI, O. S.; FERRAUDO, A. S.; CENTURION, J. F.. Análise multivariada na compactação de um latossolo vermelho cultivado com milho. Revista Brasileira de Ciência do Solo, Viçosa, v.32 n.3, p.953-961, 2008. DOI: http://dx.doi.org/10.1590/s0100-06832008000300005

GIROTTO, A. F.; ÁVILA, V. S.. Cama de aviário: análise econômica de materiais alternativos. Comunicado Técnico Embrapa Suínos e Aves, 326. Concórdia: Embrapa, 2003.

GRANGEIRO, L. C.; NEGREIROS, M. Z.; SOUZA, B. S.; AZEVÊDO, P. E.; OLIVEIRA, S. L.; MEDEIROS, M. A.. Acúmulo e exportação de nutrientes em beterraba. Ciência e Agrotecnologia, v.31 n.2, p.267-273, 2007. DOI: http://doi.org/10.1590/S1413-70542007000200001

HEYLMANN, K. K. A.; BECKER, R.; MENDES, P. M.; LUCIA, T.; CORRÊA, L. B.; CORRÊA, É. K.. Bioindicadores para determinação da estabilização da cama de frangos destinadas a adubação orgânica. In: CONGRESSO DE INICIAÇÃO CIENTÍFICA, 22. Anais. Pelotas: UFPEL, 2013.

HIGASHIKAWA, F. S.; KURTZ, C.. Manejo do solo, correção e adubação. In: MENEZES JUNIOR, F. O. G.; MARCUZZO, L. L.. Manual de boas práticas agrícolas: guia para a sustentabilidade das lavouras de cebola do estado de Santa Catarina. Florianópolis: Epagril, 2016. p.49-61.

JULIANO, P. H. G.; DIAS, T. V.; GALATI, V. C.; FERNANDES, C. M.. Aplicação de torta de filtro e cama de frango na produção de tomate de mesa. Scientific Electronic Archives, v.12, n.6, p.5-12, 2019. DOI:

http://dx.doi.org/10.36560/1262019869

KRAUSE, M. R.; MONACO, P. A. V. L.; HADDADE, I. R.; MENEGHELLI, L. A. M.; SOUZA, T. D.. Aproveitamento de resíduos agrícolas na composição de substratos para produção de mudas de tomateiro. Horticultura Brasileira, v.35, n.2, p.305-310, 2017. DOI:

http://dx.doi.org/10.1590/s0102-053620170224

LANDGRAF, M. D.; MESSIAS, R. A.; REZENDE, M. O. O.. A importância ambiental da vermicompostagem: vantagens $\mathrm{e}$ aplicações. São Carlos: Rima, 2005.

LANNA, N. B. L.; SILVA, P. N. L.; COLOMBARI, L. F.; CORRÊA C. V.; CARDOSO, A. I. I.. Efeito residual da adubação com composto orgânico na produção de rabanete. Horticultura Brasileira, Vitória da Conquista, v.36, n.1, p.47-53, 2018. DOI: http://dx.doi.org/10.1590/s0102-053620180108

LINHARES, P. C. F.; PEREIRA, M. F. S.; OLIVEIRA, B. S.; HENRIQUES, G. P. S. A.; MARACAJA, P. B.. Produtividade de rabanete em sistema orgânico de produção. Revista Verde, Mossoró, v.5, n.5, p.94-101, 2010.

MAIA, P. M. E.; AROUCHA, E. M. M.; SILVA, M. O. P.; SILVA, R. C. P.; OLIVEIRA, F. A.. Desenvolvimento e qualidade do rabanete sob diferentes fontes de potássio. Revista Verde, Mossoró, v.6, n.1, p.148-153, 2011.

MALAVOLTA, E.; PIMENTEL-GOMES, F.; ALCARDE, J. C.. Adubos e adubações. São Paulo: Nobel, 2002.
MARQUES, C. S.; GUIMARÃES, P. V. P.; SMIDERLE, O. J.; SOUSA, R. C. P.. Morfometria e crescimento inicial de Copaifera pubiflora exposta à terra vegetal produzida em compostagem. Acta Brasiliensis, v.2, n.1, p.1-5, 2018. DOI: http://dx.doi.org/10.22571/2526-433866

MENEZES, R. S. C.; SAMPAIO, E. V. S. B.; SILVEIRA, L. M.; TIESSEN, H.; SALCEDO, I. H.. Produção de batatinha com incorporação de esterco e/ou crotalária no Agreste paraibano. In: SILVEIRA, L.; PETERSEN, P.; SABOURIN, E.. Agricultura familiar e agroecologia no Semiárido: avanços a partir do agreste da Paraíba. Rio de Janeiro: AS-PTA, 2008. p.251-257.

MIOTTO, D. M. M.; MACHADO, N. R. C. F.. Purificação do subproduto do processo de extração de esteviosídeo. Ciência e Tecnologia de Alimentos, v.24, n.1, p.146-150, 2004. DOI: http://dx.doi.org/10.1590/s010120612004000100026

NURZYŃSKA-WIERDAK, R.; KATARZYNA, D.; EWA, R.; ZBIGNIEW, J.. Effects of nitrogen and potassium fertilization on growth, yield and chemical composition of garden rocket. Acta Scientiarum Polonorum Hortorum Cultus, v.11, n.2, p.289-300, 2012.

PANDEY, R.; ZINTA, G.; ABDELGAWAD, H.; AHMAD, A.; JAIN, V.; JANSSENS, I. A.. Physiological and molecular alterations in plants exposed to high [CO2] under phosphorus stress. Biotechnology Advances, New York, v.33, n.3-4, p.303-316, 2015. DOI:

http://dx.doi.org/10.1016/j.biotechadv.2015.03.011

PELÁ, A.; SILVA JUNIOR, G. S.; SILVA, R. C. D.; SILVA, C. S.; PELÁ, G. M.. Produção e teor de nitrato em rúcula sob adubação orgânica com cama de frango e esterco bovino. Revista Verde de Agroecologia e desenvolvimento Sustentável, v.12, n.1, p.48-54, 2017. DOI: http://dx.doi.org/10.18378/rvads.v12i1.4476

PENTEADO, S. R.. Manual prático de agricultura orgânica Campinas: Autor, 2007.

PIRES, A. M. M.; MATTIAZZO, M. E.. Avaliação da viabilidade do uso de resíduos na agricultura. Circular Técnica Embrapa, Jaguariúna v.1, n.19, p.1-9, 2008.

RODRIGUES, J. F.; REIS, J. M. R.; REIS, M. A.. Utilização de estercos em substituição a adubação mineral na cultura do rabanete. Revista Trópica: Ciências Agrárias e Biológicas, v.7, n.2, p.160-168, 2013. DOI: http://dx.doi.org/10.0000/rtcab.v7i2.1110

SILVA, A. F. A.; SOUZA, E. G. F.; JÚNIOR, A. P. B.; NETO, F. B.; SILVEIRA, L. M.. Desempenho agronômico do rabanete adubado com Calotropis procera (Ait.) R. Br. em duas épocas de cultivo. Revista Ciência Agronômica, Fortaleza, v.48, n.2, p.328-336, 2017. DOI: http://dx.doi.org/10.5935/1806$\underline{6690.20170038}$

SILVA, A. F. A.; SOUZA, E. G. F.; SANTOS, M. G.; BARROS JUNIOR, A. P.; BEZERRA, F.; SILVEIRA, L. M.. Rentabilidade do rabanete adubado com flor de seda em duas épocas de cultivo no semiárido de Pernambuco. Revista de Ciências Agrárias, v.58, n.2, p.198-207, 2015. DOI:

http://dx.doi.org/10.4322/rca.1761 
TRANI, P. E.; TERRA, M. M.; TECCHIO, M. A.; TEIXEIRA, L. A. J.; HANASIRO, J.. Adubação Orgânica de Hortaliças e Frutíferas. Campinas: IAC, 2013.
ZÖRB, C.; SENBAYRAM, M.; PEITER, E.. Potassium in agriculture: status and perspectives. Journal Of Plant Physiology, v.171, n.9, p.656-669, 2014. DOI: http://dx.doi.org/10.1016/i.jplph.2013.08.008

A CBPC - Companhia Brasileira de Produção Científica (CNPJ: 11.221.422/0001-03) detém os direitos materiais desta publicação. Os direitos referem-se à publicação do trabalho em qualquer parte do mundo, incluindo os direitos às renovações, expansões e disseminações da contribuição, bem como outros direitos subsidiários. Todos os trabalhos publicados eletronicamente poderão posteriormente ser publicados em coletâneas impressas sob coordenação da Sustenere Publishing, da Companhia Brasileira de Produção Científica e seus parceiros autorizados. Os (as) autores (as) preservam os direitos autorais, mas não têm permissão para a publicação da contribuição em outro meio, impresso ou digital, em português ou em tradução. 